\title{
Microstructure Investigation of Radiochromic Dosimetry Films Using Positron Annihilation Lifetime Spectroscopy
}

\author{
N.T.S. Albogamy ${ }^{1}$, T. Sharshar ${ }^{2,3}$, A. Aydarous ${ }^{2 *}$ \\ ${ }^{1}$ Physics Department, Faculty of Applied Medical Sciences, Taif University-Tarabah Branch, Tarabah, Saudi \\ Arabia \\ ${ }^{2}$ Physics Department, Faculty of Science, Taif University, Al-Hawiah, Taif, P.O. Box 888, Zip Code 21974, \\ Saudi Arabia \\ ${ }^{3}$ Physics Department, Faculty of Science, Kafrelsheikh University, Kafr El-Sheikh, Egypt
}

\begin{abstract}
Gafchromic $M D-55$ and $H D-V 2$ films wereirradiated with gamma doses in the range 0-80 Gy and 0 $2000 \mathrm{~Gy}$, respectively. The microstructure changes of these films have been probedusing positron annihilation lifetime (PAL) spectroscopy for the first time. The dependence of the PAL parametersupon the microstructure of the active component of both films such as its polymerizationdegreewasdiscussed. A possible correlationbetween the formation probability of ortho-positronium and the opticaldensity of radiochromic films type $H D-V 2$ wasobserved. The applicability of the PAL technique to study the physical changes occurred in the active layer of the radiochromic films during the solid-state polymerizationreactioncaused by $\gamma$ irradiation wasdemonstrated.
\end{abstract}

Keywords: Radiation effects, $M D-55$ and $H D-V 2$ radiochromic films, Positron annihilation life time spectros copy, $\gamma$ irradiation

\section{Introduction}

Radiochromic films are a type of films used in radiotherapy as a dosimeter. These radiochromic dosimeters have also been appliedin many fields such as environmental studies and industrial applications. The color of these films is changed in proportion to the absorbed dose caused by film exposure toionizing radiation. The film color changes to a dark blueby a radiochromic reaction (solid-state polymerization). The color change of the film active layer is mainly due to the significant chemical modifications of this layer caused by its exposure to radiation [1,2]. This color change of the active layer of the film is used to quantify the absorbed dose.

The radiochromic films are self-developing in response to radiation without chemical, physical and thermal treatments pre- and/or post-irradiation. On the other hand, these films are affected by certain types of light, such as sunlight and other UV sources, especially if the film is exposed for a long periods of time. The dependence of thecolor change of radiochromic filmson the energy of incident radiation is relatively small.The radiochromic films are also dose-rate independent[1].The absorbed dose from high-energy photons can be measured by the radiochromic films types MD-55 and HD-V2. The detailed of the configurations, technical features, specifications and performance data of both films are reported in Refs [3,4].

Positron annihilation spectroscopy (PAS) is widely used to study the structural changesand electronic distribution of various types of materials. The PAS is based on the detection of annihilation radiation after implantation of positronsin studied samples.The positron annihilation lifetime (PAL)is one of PAS techniques that used to characterize thedefects and open volumes of solid samples[5-12].The implanted positrons in solids probe the electronic environment around their annihilation sites.Some of the incident positrons annihilate through a positronium (Ps), the bound state of a positron and an electron in polymeric materials. The Ps has two states: the para-Ps (p-Ps) and ortho-Ps (o-Ps) with anti-parallel and parallel spins of the positron and electron, respectively. The PAS is a preferred probeforpolymeric materials as the Ps annihilates in the free volume holes.

Several studies were carried out to investigate the factors such as the photon energy affect the sensitivity of the radiochromic films.Recently, the positron annihilation Doppler broadening (PADB) spectroscopy was applied to investigate the defect structure of the active component of the $\gamma$-irradiated MD-55 and HD-V2 radiochromic films [13]. To clarify and confirm the applicability of PAS techniques for investigation of the physical changes in the active component of radiochromic films due to $\gamma$ irradiation,thiswork therefore aims toprobethe structural changes of the active component of the $\gamma$-irradiated MD-55 and HD-V2 radiochromic films using the PAL technique. The correlation between PAL parameters and theoptical properties of the MD-55 andHD-V2 films can also be examined. 


\section{Experimental methods}

Radiochromic films types MD-55 and newly released HD-V2, ISP Technologies Inc. Dosimetry Media, were used. Fourand eight sets (with ten pieces for each set) of MD-55 and HD-V2 films were prepared, respectively, with an area of $2 \times 2 \mathrm{~cm}^{2}$ for each piece. Three setsof MD-55 films were given $\gamma$ doses of 20, 40 and $80 \mathrm{~Gy}$ for each.Seven sets of HD-V2 films wereirradiated with gamma doses of 50, 100, 500, 800, 1000, 1500 and $2000 \mathrm{~Gy}$. Both films types MD-55 and HD-V2 were irradiated at room temperature using ${ }^{137} \mathrm{Cs}$ and ${ }^{60} \mathrm{Co}$ sources with dose rates of 0.4615 and 100 Gy min-1, respectively. The detailed information about the preparation and irradiation of the samples of both films are reported in Ref.[13].

The PAL measurements were performed using a fast-fast coincidence spectrometer[14]. This spectrometer consists of two Bicron BC-418 plastic scintillation detectors coupled with Ortecelectronic modules. The lifetime measuredspectra were acquired using an Ortec 919 multichannel analyzer. A $10 \mu \mathrm{Ci}$ positron source was prepared using a droplet of ${ }^{22} \mathrm{NaCl}$ solution dried onto two identical Kapton foils $(7.5 \mu \mathrm{m}$ thick), which were afterward glued by epoxy glue. Four pieces of each film were placed in contact with each side of the ${ }^{22} \mathrm{Na}$ source to arrange the source and sample in a sandwich like configuration. ThePAL measurements were carried out in air at room temperature. More than one million counts were accumulated for each spectrum and each sample was measured three times. The time resolution of the spectrometer, measured with ${ }^{60} \mathrm{Co}$ source at ${ }^{22} \mathrm{Na}$ energy window settings, was $\sim 320 \mathrm{ps}$ (full-width at half maximum). The positron annihilation lifetime is measured experimentally as the time interval between the $1274.5 \mathrm{keV} \gamma$ ray emitted by the ${ }^{22} \mathrm{Na}$ radioisotope and one of the annihilation radiations.

The PAL spectra were analyzed using a computer program LT [15] with $12 \%$ correction for positrons annihilated in the Kapton. The lifetime spectra of the investigated samples were decomposed into three components representing the p-Ps $\left(\tau_{l}=125 \mathrm{ps}\right)$, free and bound positrons $\left(\tau_{2}=150-500 \mathrm{ps}\right)$ and pickoff annihilation of the o-Ps in the free volume sites present in the active layers of the studied radiochromic films ( $\tau_{3}>700 \mathrm{ps}$ ). The formation probabilities of the $\tau_{1}, \tau_{2}$, and $\tau_{3}$ are $I_{1}, I_{2}$ and $I_{3}$, respectively. The lifetime spectra of the investigated samples were decomposed into three components. As the time resolution of the used PAL spectrometer $>300 \mathrm{ps}$, the data on the shortest-lived component ascribed to p-Ps somewhat unreliable. Therefore, $\tau_{l}$ was fixed at $125 \mathrm{ps}$ to reduce the scatter of the other parameters. All lifetime components were determined with the best-fitting parameter ranged from 0.99 to 1.2. The range of the experimental errors for the PAL parameters $\tau_{2}, \tau_{3}, I_{1}, I_{2}$ and $I_{3}$ determined over multiples measurements, were found to be $<5 \mathrm{ps},<21 \mathrm{ps}$, $1.3 \%, 1.2 \%$ and $0.4 \%$, respectively. The mean lifetime $\left(\tau_{m}\right)$ of positron was calculated using the following relation:

$$
\tau_{m}=\frac{\tau_{1} I_{1}+\tau_{2} I_{2}+\tau_{3} I_{3}}{I_{1}+I_{2}+I_{3}}
$$

The mean free volume, $V_{f}$, can be calculated by a simple relation given below, assuming a spherical shape for the holes[7,9,16,17]:

$V_{\mathrm{f}}=4 / 3 \pi \mathrm{R}^{3}$

where $\mathrm{R}$ is the average radius of the free volume hole. $\mathrm{R}$ is determined using a semiempirical equation with the values of $\tau_{3}[18,19]$. Furthermore, the free volume hole fraction, $f_{v}$, can be found from the empirical equation[15,20]:

$f_{\mathrm{v}}=C V_{\mathrm{f}} I_{3}$,

where $V_{f}$ is in $\AA^{3}, I^{3}$ in \%, and Cis a structural constant empirically determined to be about 0.0018 [21].

\section{Results and discussion}


The PAL parameters $\left(\tau_{2}, \tau_{3}, I_{2}, I_{3}\right.$ and $\left.\tau_{m}\right)$ as a function of $\gamma$-ray absorbed dose are shown in
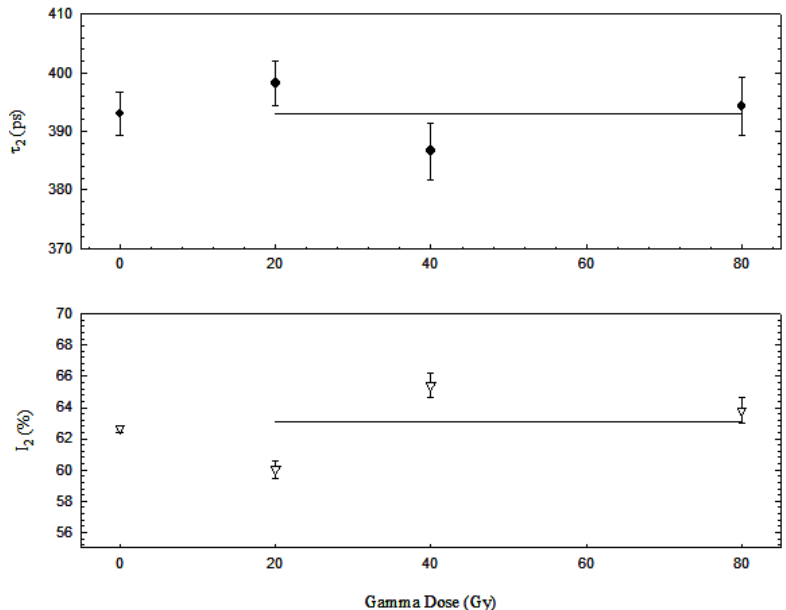

Fig. 1, Fig.2 and Fig.3 for MD-55 radiochromic films. Fig.4, Fig.5and Fig.6show the dependence of these parameters on the $\gamma$ absorbed dose in the case of HD-V2 radiochromic films. The results show that high Ps formation probability, I3, (o-Ps intensities)is observed withmean value and range of 18.45 $\pm 0.21(18.19-18.64)$ and 18.40 \pm 0.76 (17.58-19.98\%) forMD-55 and HD-V2 films, respectively. These high intensities of Ps formation are expected for polymeric materials. The calculated average radius of the free volume hole and the mean free volume of unirradiated and irradiated MD-55 and HD-V2 films are listed in

Table 1.The results indicate that the average radius of the free volume hole in MD-55 films is larger than that of HD-V2 films by 7.3\%. These results also show that the effect of $\gamma$ irradiation on the concentration of free volume holes is strong in the case of HD-V2 films as the range of $I_{3}$ values of HD-V2 films is larger than that range of the MD-55 films. This increase of the average radius of the free volume holes and the difference of the effect of $\gamma$ irradiation on the concentration of free volume holes may be due to the different internal structure and active-layer thickness of both films. The calculated free volume hole fraction as a function of $\gamma$ absorbed dose is shown in Fig.7 and Fig.8 for MD-55 and HD-V2 films, respectively. The free volume hole fraction in the MD-55 films showed an increase of $~ 24 \%$ compared with its values in HD-V2 films. An opposite behavior of the free volume hole fraction as a function of $\gamma$ absorbed dose was also observed for both films. This different behavior of both films can be explained by the previously mentioned reasons.

The mean values of the PAL parameters $\left(\tau_{2}, \tau_{3}, I_{2}\right.$ and $\left.I_{3}\right)$ and the derived PAL parameters $\left(\tau_{m}\right.$ and $\left.f_{v}\right)$

were calculated for yirradiated MD-55 films with doses 20, 40 and 80 Gy thatlie in the film dynamic dose range(2-100 Gy), see
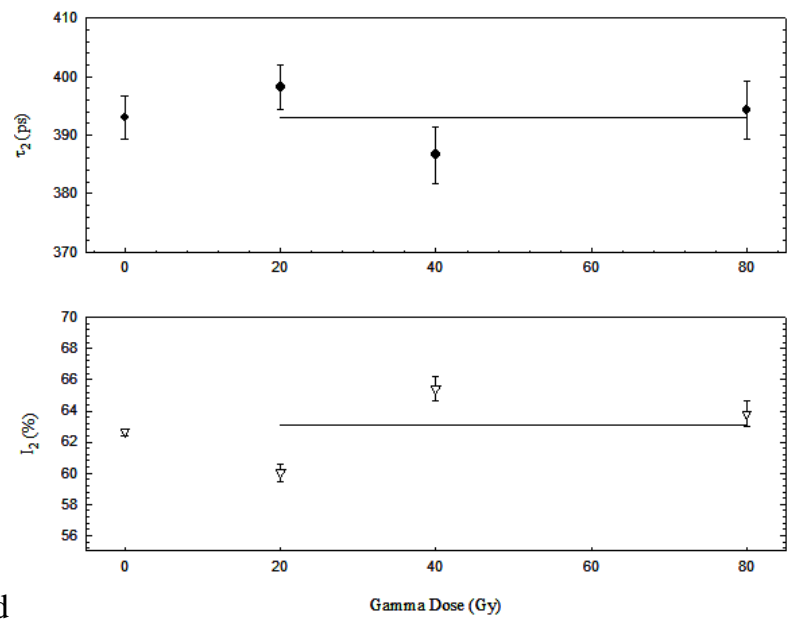

Table 2 and 
Fig. 1, Fig.2, Fig.3 and Fig.7. Themean values of thesePAL parameters are also calculated for yirradiated HD-V2 films with doses 50, 100, 500, 800 and 1000 Gy that lie in the film dynamic dose range, 10$1000 \mathrm{~Gy}$, and with doses 1500 and 2000 Gy that lie out of the film dynamic dose range, see

Table 2and Fig.4, Fig.5, Fig.6 and Fig.8.The results show that the meanvalues of PAL parametersfor MD-55 and HD-V2 films irradiated with $\gamma$ dose withintheir dynamic dose range are almost constantwith a tendency of a little decrease compared with those values of unirradiated films. The mean value of the $f_{v}$ for irradiated HD-V2 film with $\gamma$ dose in the film dynamic range is almost equalto the value of the unirradiated film, although the observed increase of the $f_{v}$ values with $\gamma$ irradiation with doses from 50 to 800 Gy. This observed increasein the $f_{v}$ values may be due to the increasing of the freevolume holeconcentration, I3, caused by the $\gamma$ irradiation (see Fig.5). In the case of HD-V2 film irradiated with $\gamma$ dose out of its dynamic dose range, the mean values of PAL parameters show a clear decrease compared with the value of the unirradiated film. The observed stability with slow decrease tendency of most PAL parameters for the irradiated MD-55 and HD-V2 films with doses in the range of film dynamic dosemay be due tothe polymerization of the active component of these films that dependent on the absorbed dose. This polymerization process changes the microstructure of the film active component by changing various structure parameters such as electron density distribution, size and concentration of free volume and polymer-monomer mixture ratio.Therefore, the stability of the mean values of the PAL parameters is mainly due to the scattered decreasing or increasing the values of the structure parameters. On the other hand, the observed clear decrease for the HD-V2 films irradiated with $\gamma$ dose greater than their dynamic dose range may be due to the photo-degradation of the formed polymer structure of the active component. The scattered change of most PAL parameters for irradiated HD-V2 films at the beginning of its dynamic dose range indicates the strongeffectof $\gamma$ irradiation on this type of radiochromic films at itsstart region oftheir dynamic dose range. This scattered behavior may be due to the thin thickness of the film active layer $(\sim 8 \mu \mathrm{m})$. Therefore, the results of this work suggested that the performance of the HD-V2 films can be enhanced by increasing the thickness of the film active layer, by adding another component to the active layer or by changing the proportions of the atomic constituents of this sensor layer with keeping the film technical feature of near tissue equivalent. The variation of $f_{v}$ parameter with the optical density of the unirradiated and irradiated HD-V2 films, reported in Ref. [13], is shown in 


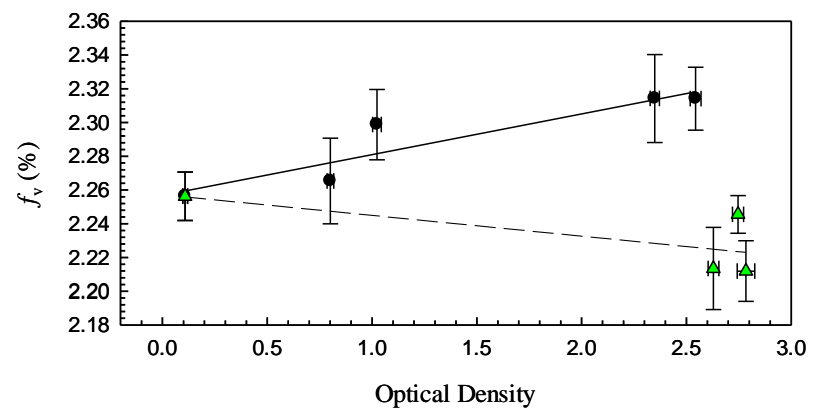

Fig.9. Similar behavior was observed for the variation of $f_{v}$ parameter as a function of gamma dose. The results indicate that a strong positivecorrelation with the linear coefficient parameter, $r=0.92$, was observed between the $f_{v}$ and OD values for $\gamma$-irradiated HD-V2 films with doses<1000 Gy. This strong correlation may be due to the observed linear increase of the freevolume hole fraction with increasing of the $\gamma$ dose (seeFig.8). A weak negative correlation with $\mathrm{r}=0.71$ between the $f_{v}$ and OD values for $\gamma$-irradiated HD-V2 films with doses $\geq 1000$ Gy was observed. This negative correlation may be due to the photo-degradation of the formed polymer structure of the active component and the decrease of the free volume hole fraction (see Fig.8). Therefore, it can be concluded that the derived PAL parameter $f_{v}$ can be used to investigate the response behavior and to define the limit of the dynamic dose range of the radiochromic films. The importance of $f_{v}$ parameter comes mainly from its dependence on the Ps formation (see the last paragraph in section 2) that trapped in the free volume holes of polymeric materials.

\section{Conclusion}

The PAL techniquewas used for the first time to probe the defects and structural changes of the $\gamma$ irradiated MD-55 and HD-V2 radiochromic films with absorbed doses ranges of 0-80 and 0-2000 Gy, respectively. The results of this work indicate that the behavior of the PAL parameters was successfully related to the microstructures of the active (radiosensitive) layers of MD-55 and HD-V2 films. One of these parameters such as $f v$ that evaluate the free volume size and concentrationcan be used to investigate the response behavior and to define the limit of the dynamic dose range of the radiochromic films. A good linear correlation was found between the $f v$ parameter and the OD values for HD-V2 film. A proposal is suggested to enhance the HD-V2 film performance through changing the film thickness and structure. Finally, it can be concluded that the PAS techniques are a powerful tool to probe the microstructure of the $\gamma$ irradiated radiochromic films.

\section{References}

[1]. Devic, S., 2011. Radiochromic film dosimetry: past, present, and future.Physica medica, 27, 122-134.

[2]. Niroomand-Rad, A., Blackwell, C.R., Coursey, B.M., Gall, K.P., Galvin, J.M., McLaughlin, W.L., Meigooni, A.S., Nath, R., Rodgers, J.E. and Soares, C.G., 1998. Radiochromic film dosimetry: recommendations of AAPM radiation therapy committee task group 55.Medical physics, 25, 2093-2115.

[3]. International Specialty Products, 2012.

[4]. International Specialty Products, 2010.

[5]. Schrader, D. and Jean, Y., 1988. New York: Elsevier.

[6]. Jean, Y., Dupasquier, A. and Mills Jr, A., 1995. Positron spectroscopy of solids.IOS, Amsterdam, 563.

[7]. Marques-Netto, A., Carvalho, C.d.F., de Carvalho, C.A., Sinisterra, R.D., Brito, H.F.d. and Machado, J.C., 2001. Positronium formation in lanthanide (III) trifluoroacetate with 3-picoline-N-oxide compounds.Chemical physics letters, 333, $371-374$.

[8]. Abdel-Hady, E. and Mohamed, H.F., 2002. Microstructure changes of poly (vinyl chloride) investigated by positron annihilation techniques.Polymer degradation and stability, 77, 449-456.

[9]. Jean, Y., Mallon, P. and Schrader, D.M., 2003. Principles and applications of positron \& positronium chemistry. World Scientific Publishing Company Incorporated.

[10]. He, C., Suzuki, T., Shantarovich, V., Djourelov, N., Kondo, K. and Ito, Y., 2004. Positron annihilation studies of hyper-cross-linked polystyrenes.Chemical physics, 303, 219-226.

[11]. Abdel-Rahman, M., Abo-Elsoud, M., Eissa, M., Kamel, N., Lotfy, Y. and Badawi, E., 2006. Positron Annihilation Line Shape Parameters for CR-39 Irradiated by Different Alpha-Particle Doses.Progress in Physics, 3, 91.

[12]. Lounis-Mokrani, Z., Guittoum, A., Imatoukene, D., Aitziane, M., Badreddine, A. and Mebhah, M., 2013. Characterisation of proton irradiated CR-39 detector using positron annihilation lifetime spectroscopy.Radiation Measurements, 50, 26-30.

[13]. Albogamy, N., Sharshar, T. and Aydarous, A., 2015. The use of positron annihilation Doppler broadening spectroscopy in the characterization of radiochromic dosimetry films.Radiation Measurements, 75, 1-5.

[14]. Sharshar, T. and Hussein, M., 2005. An optimization of energy window settings for positron annihilation lifetime spectrometers.Nuclear Instruments and Methods in Physics Research Section A: Accelerators, Spectrometers, Detectors and Associated Equipment, 546, 584-590.

[15]. Kansy, J., 1996. Microcomputer program for analysis of positron annihilation lifetime spectra.Nuclear Instruments and Methods in Physics Research Section A: Accelerators, Spectrometers, Detectors and Associated Equipment, 374, $235-244$.

[16]. Nakanishi, H., Jean, Y., Smith, E. and Sandreczki, T., 1989. Positronium formation at free-volume sites in the amorphous regions of semicrystalline PEEK.Journal of Polymer Science Part B: Polymer Physics, 27, 1419-1424. 
[17]. Porto, A., Silva, G.G. and Magalhaes, W., 1999. Free volume-size dependence on temperature and average molecular-weight in poly (ethylene oxide) determined by positron annihilation lifetime spectroscopy.Journal of Polymer Science Part B Polymer Physics, 37, 219-226.

[18]. Eldrup, M., Lightbody, D. and Sherwood, J., 1981. The temperature dependence of positron lifetimes in solid pivalic acid.Chemical Physics, 63, 51-58.

[19]. Ito, K., Ujihira, Y., Yamashita, T. and Horie, K., 1999. Free-volume change in volume phase transition of polyacrylamide gel as studied by positron annihilation: Salt dependence.Journal of Polymer Science Part B: Polymer Physics, 37, $2634-2641$.

[20]. Schmidt, M. and Maurer, F., 2000. Relation between free-volume quantities from PVT-EOS analysis and PALS.Polymer, 41, 84198424 .

[21]. Kumar, R. and Prasad, R., 2007. Ion induced modification in free volume in PN-6 and PES polymers by positron annihilation lifetime studies.Nuclear Instruments and Methods in Physics Research Section B: Beam Interactions with Materials and Atoms, $256,238-242$.

Table 1. The calculated average radius of the free volume hole and the mean free volume for unirradiated and irradiated MD-55 and HD-V2 radiochromic films.

\begin{tabular}{|l|l|l|}
\hline Gamma-rayabsorbed dose (Gy) & $\mathrm{R}(\mathrm{nm})$ & $V_{f}\left(\mathrm{~nm}^{3}\right)$ \\
\hline MD-55 films & $0.2738 \pm 0.0027$ & $0.0860 \pm 0.0008$ \\
\hline 0 & $0.2729 \pm 0.0018$ & $0.0852 \pm 0.0006$ \\
\hline 20 & $0.2709 \pm 0.0028$ & $0.0833 \pm 0.0009$ \\
\hline 40 & $0.2751 \pm 0.0031$ & $0.0873 \pm 0.0010$ \\
\hline 80 & \multicolumn{3}{|l|}{} \\
\hline HD-V2 films & $0.2552 \pm 0.0011$ & $0.0696 \pm 0.0003$ \\
\hline 0 & $0.2533 \pm 0.0028$ & $0.0681 \pm 0.0007$ \\
\hline 50 & $0.2480 \pm 0.0022$ & $0.0639 \pm 0.0006$ \\
\hline 100 & $0.2535 \pm 0.0026$ & $0.0682 \pm 0.0007$ \\
\hline 500 & $0.2575 \pm 0.0018$ & $0.0716 \pm 0.0005$ \\
\hline 800 & $0.2511 \pm 0.0025$ & $0.0663 \pm 0.0007$ \\
\hline 1000 & $0.2556 \pm 0.0011$ & $0.0700 \pm 0.0003$ \\
\hline 1500 & $0.2555 \pm 0.0018$ & $0.0699 \pm 0.0005$ \\
\hline 2000 & & \\
\hline
\end{tabular}

Table 2. Themean values of the PAL parameters $\left(\tau_{2}, \tau_{3}, \mathrm{I}_{2}\right.$ and $\left.\mathrm{I}_{3}\right)$ and the derived PAL parameters $\left(\tau_{\mathrm{m}}\right.$ and $\left.f_{v}\right)$ for unirradiated and irradiated MD-55 and HD-V2 radiochromic films.

\begin{tabular}{|l|l|l|l|l|l|l|}
\hline \multirow{2}{*}{$\begin{array}{l}\text { PAL } \\
\text { Parameter }\end{array}$} & \multicolumn{2}{|l|}{ Mean Values } & \multicolumn{2}{l|}{$\begin{array}{l}\text { Irradiated with dose in the } \\
\text { dynamic range }\end{array}$} & $\begin{array}{l}\text { Irradiated with dose > the dynamic dose } \\
\text { range }\end{array}$ \\
\cline { 2 - 7 } & Unirradiated & HD-V2 & MD-55 & HD-V2 & MD-55 & HD-V2 \\
\cline { 2 - 7 } & $393 \pm 2$ & $378 \pm 3$ & $393 \pm 3$ & $368 \pm 8$ & - & $378 \pm 5$ \\
\hline$\tau_{2}(\mathrm{ps})$ & $1851 \pm 8$ & $1670 \pm 5$ & $1843 \pm 10$ & $1647 \pm 10$ & - & $1674 \pm 1$ \\
\hline$\tau_{3}(\%)$ & $62.6 \pm 0.1$ & $61.6 \pm 0.7$ & $63.1 \pm 0.7$ & $63.0 \pm 1.5$ & - & $58.6 \pm 1.5$ \\
\hline$I_{3}(\%)$ & $18.6 \pm 0.1$ & $18.0 \pm 0.1$ & $18.4 \pm 0.1$ & $18.8 \pm 0.5$ & - & $17.7 \pm 0.1$ \\
\hline$\tau_{m}(\mathrm{ps})$ & $615 \pm 5$ & $566 \pm 5$ & $610 \pm 7$ & $559 \pm 7$ & - & $547 \pm 1$ \\
\hline$f_{v}(\%)$ & $2.89 \pm 0.01$ & $2.26 \pm 0.01$ & $2.82 \pm 0.02$ & $2.28 \pm 0.03$ & - & $2.23 \pm 0.02$ \\
\hline
\end{tabular}



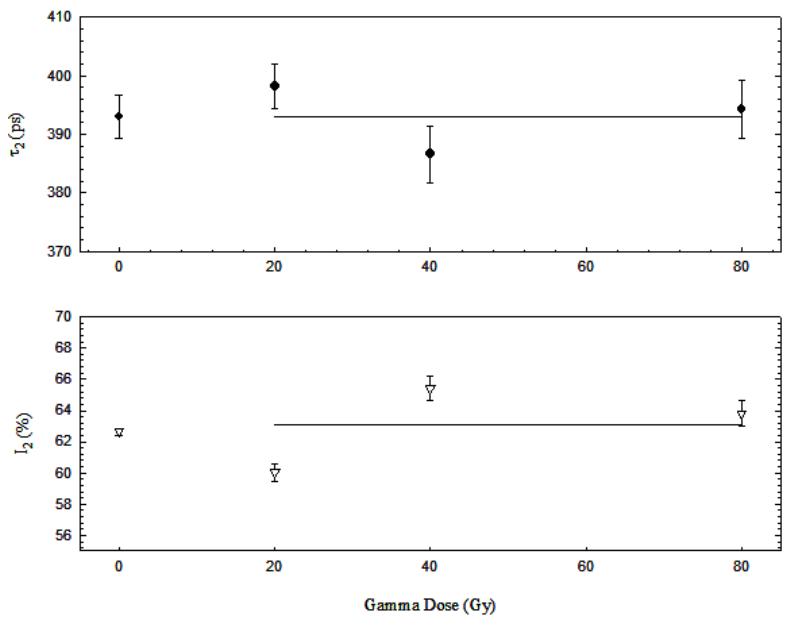

Fig. 1:The lifetime component $\tau_{2}$ and its intensity $I_{2}$ as a function of $\gamma$ dose forthe MD-55Gafchromic film. The solid line represents the mean value of $\tau_{2}$ and $\mathrm{I}_{2}$ for the $\gamma$ dose within the film dynamic dose range (20, 40 and 80 Gy).
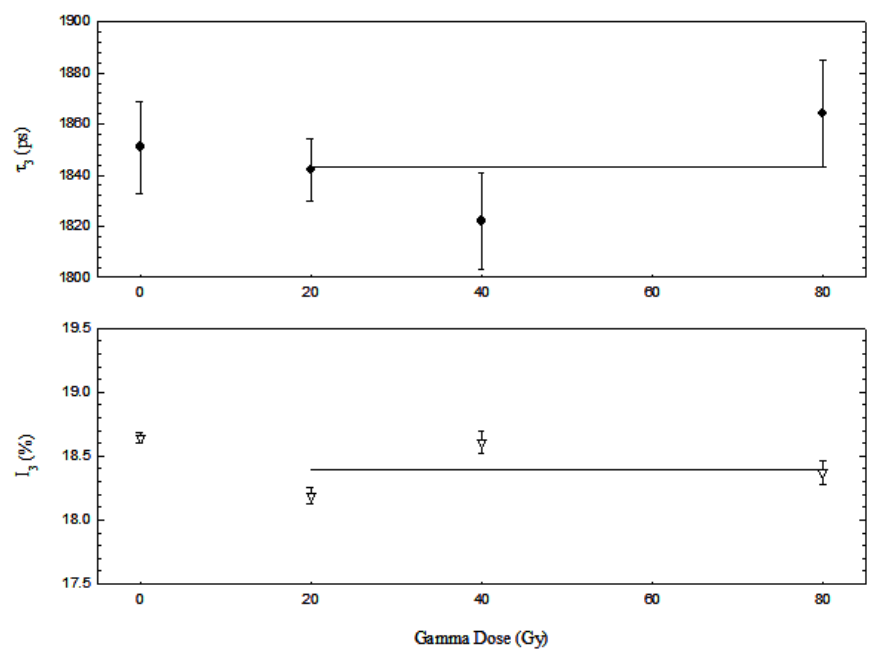

Fig.2: The lifetime component $\tau_{3}$ and its intensity $\mathrm{I}_{3}$ as a function of $\gamma$ dose for theMD-55Gafchromic film. The solid line represents the mean value of $\tau_{3}$ and $\mathrm{I}_{3}$ for the $\gamma$ dose within the film dynamic dose range (20, 40 and 80 Gy).

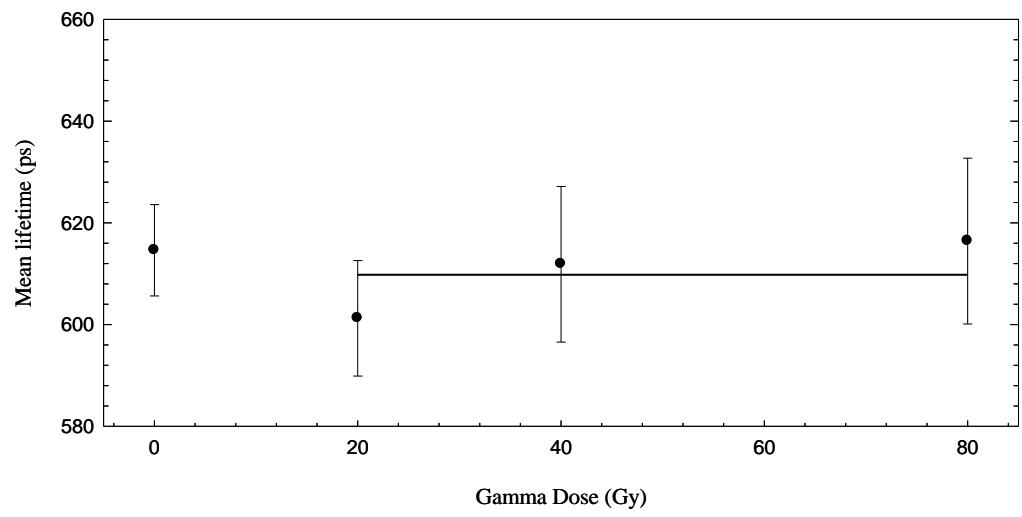

Fig.3:The mean lifetime as a function of $\gamma$ dose for the MD-55Gafchromic film.The solid line represents the mean value of $\tau_{\mathrm{m}}$ for the $\gamma$ dose within the film dynamic dose range (20, 40 and $\left.80 \mathrm{~Gy}\right)$. 

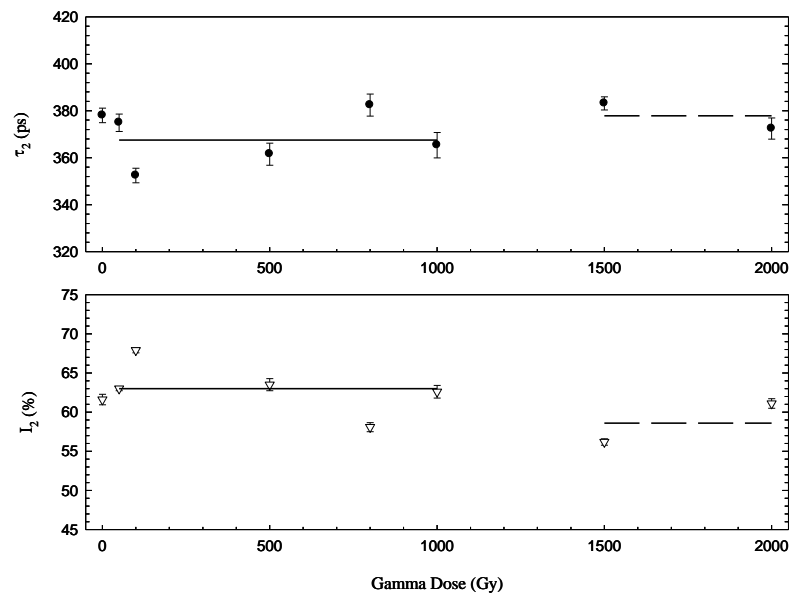

Fig.4: The lifetime component $\tau_{2}$ and its intensity $I_{2}$ as a function of $\gamma$ dose for theHD-V2Gafchromic film. The solid and dashed lines represent the mean values of $\tau_{2}$ and $\mathrm{I}_{2}$ for the $\gamma$ dose within the film dynamic dose range

$(50,100,500,800$ and $1000 \mathrm{~Gy})$ and for $\gamma$ dose greater than the dynamic dose range (1500 and $2000 \mathrm{~Gy})$, respectively.
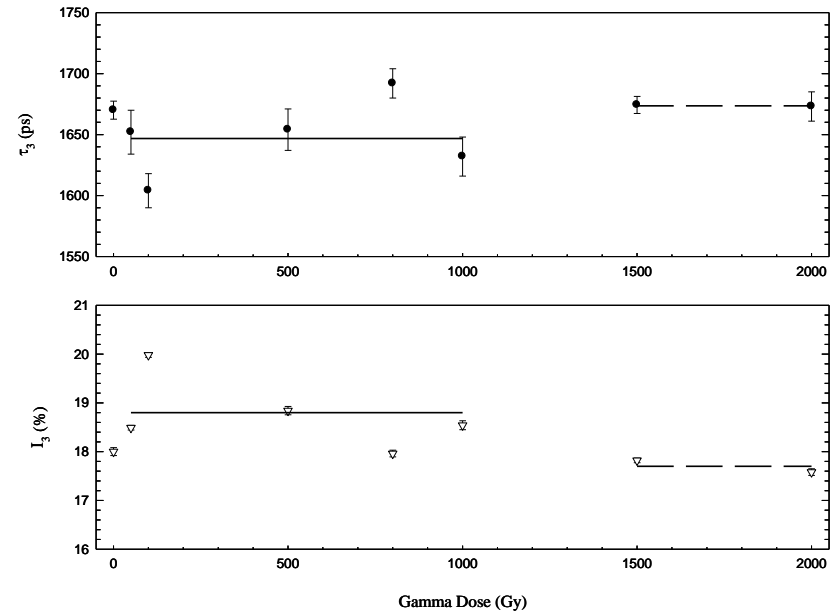

Fig.5: The lifetime component $\tau_{3}$ and its intensity $\mathrm{I}_{3}$ as a function of $\gamma$ dose for theHD-V2Gafchromic film. The solid and dashed lines represent the mean values of $\tau_{3}$ and $\mathrm{I}_{3}$ for the $\gamma$ dose within the film dynamic dose range

$(50,100,500,800$ and $1000 \mathrm{~Gy})$ and for $\gamma$ dose greater than the dynamic dose range (1500 and $2000 \mathrm{~Gy})$, respectively.

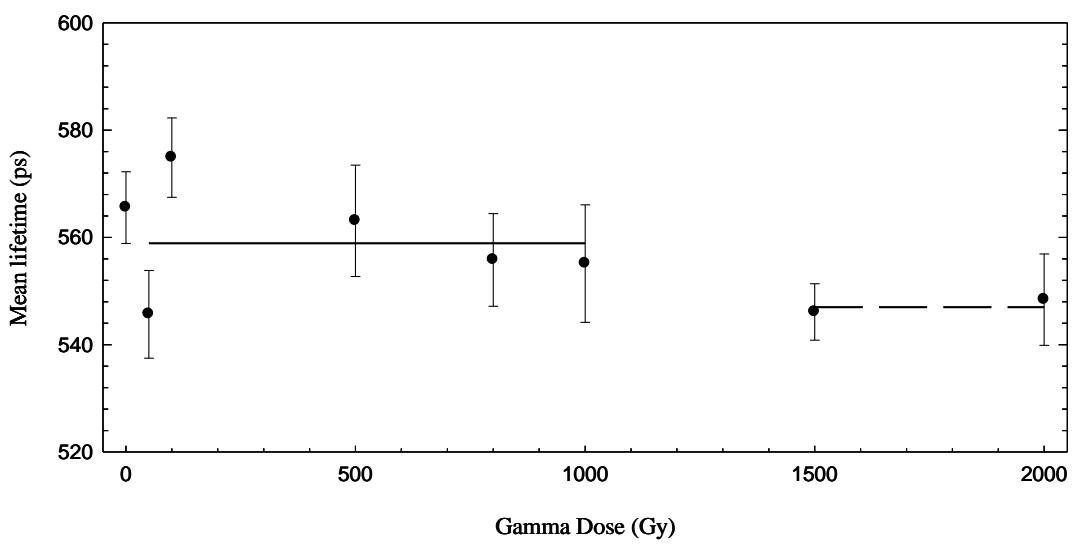

Fig.6: The mean lifetime as a function of $\gamma$ dose forthe HD-V2Gafchromic film. The solid and dashed lines represent the mean values of $\tau_{\mathrm{m}}$ for the $\gamma$ dose within the film dynamic dose range $(50,100,500,800$ and 1000

Gy) and for $\gamma$ dose greater than the dynamic dose range (1500 and $2000 \mathrm{~Gy}$ ), respectively. 


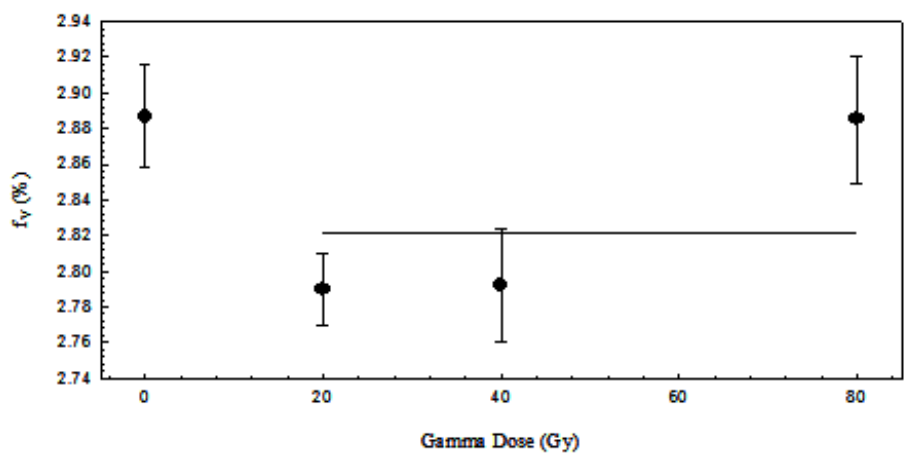

Fig.7:The free volume hole fraction $\left(f_{\mathrm{v}}\right)$ as a function of $\gamma$ dose for theMD-55Gafchromic film. The solid line represents the mean value of $f_{\mathrm{v}}$ for the $\gamma$ dose within the film dynamic dose range (20,40 and $\left.80 \mathrm{~Gy}\right)$.

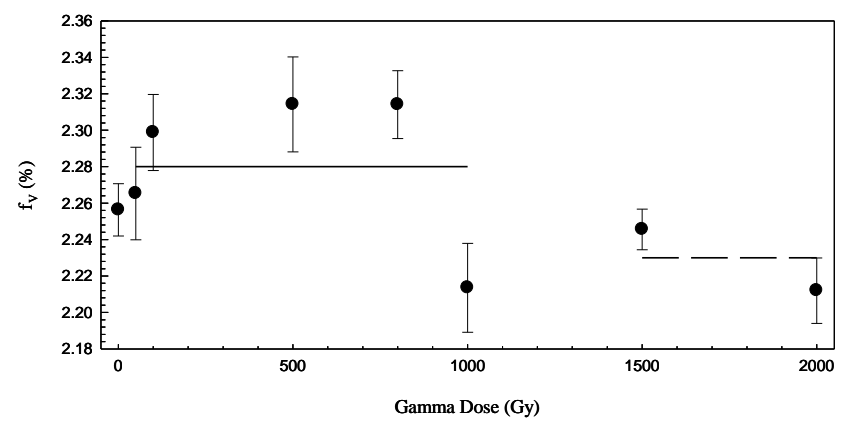

Fig.8:The free volume hole fraction $\left(f_{\mathrm{v}}\right)$ as a function of $\gamma$ dose forthe HD-V2Gafchromic film. The solid and dashed lines represent the mean values of $f_{\mathrm{v}}$ for the $\gamma$ dose within the film dynamic dose range $(50,100,500,800$ and $1000 \mathrm{~Gy}$ ) and for $\gamma$ dose greater than the dynamic dose range (1500 and $2000 \mathrm{~Gy}$ ), respectively.

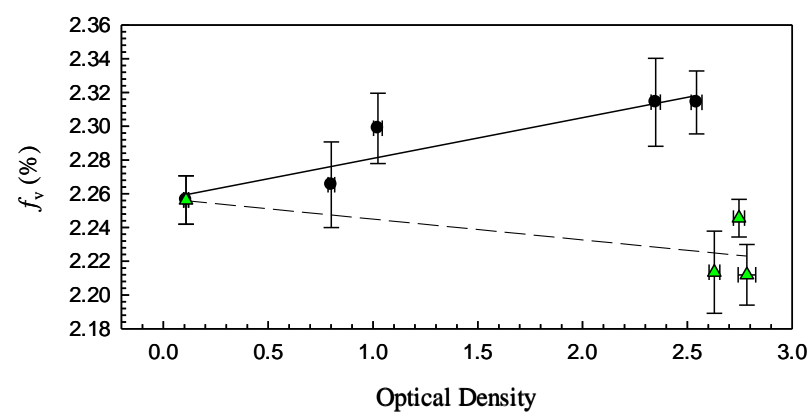

Fig.9:The PAL parameter $f_{v}$ as a function of the optical density for the HD-V2 films (taken from Ref. 7). The solid circles for unirradiated and irradiated films with $\gamma$ dose of 50, 100,500 and $800 \mathrm{~Gy}$. The solid triangles for unirradiated and irradiated films with $\gamma$ dose of 1000, 1500 and $2000 \mathrm{~Gy}$. The solid line is a linear fitting of the experimental data with $\gamma$ dose $<1000$ Gy (solid circles) and the dashed line is a linear fitting of the experimental data with unirradiated and $\gamma$ irradiated dose $\geq 1000 \mathrm{~Gy}$ (solid triangles). 